\title{
The efficacy and safety of different doses of calcitriol combined with neutral phosphate in X-linked hypophosphatemia: a prospective study
}

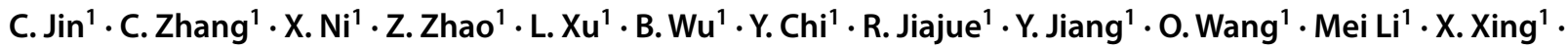 \\ X. Meng ${ }^{1} \cdot$ W. Xia ${ }^{1}$ (i)
}

Received: 7 June 2021 / Accepted: 19 October 2021 / Published online: 27 January 2022

(C) The Author(s) 2022

\begin{abstract}
Summary The present study was the first prospective cohort evaluated the efficacy and safety of different doses of calcitriol in XLH children. The results suggested that a dose of $40 \mathrm{ng} / \mathrm{kg} /$ day calcitriol, compared with $20 \mathrm{ng} / \mathrm{kg} / \mathrm{day}$, was more effective in relieving the rickets, with similar safety outcomes. Further investigations were expected to set more dose groups. Introduction Dose recommended for calcitriol in X-linked hypophosphatemia (XLH) varies in different studies. Therefore, we aimed to compare the efficacy as well as the safety of $20 \mathrm{ng} / \mathrm{kg} / \mathrm{d}$ and $40 \mathrm{ng} / \mathrm{kg} / \mathrm{d}$ calcitriol in Chinese XLH pediatrics population.

Methods A 2-year, randomized, open-label, prospective study recruited $68 \mathrm{XLH}$ children, which were randomized to receive either $40 \mathrm{ng} / \mathrm{kg} / \mathrm{day}$ or $20 \mathrm{ng} / \mathrm{kg} /$ day calcitriol. Efficacy endpoints were the total Thacher ricket severity score (RSS) change from baseline to month 12 and 24, the difference in serum TALP level, fasting serum phosphate level, body height Z-score, and frequency of dental abscess. Safety assessments were done using renal ultrasound nephrocalcinosis grades (0-4), fasting serum and $24 \mathrm{~h}$ urine calcium level, and the occurrence of hyperparathyroidism.

Results The decrease in the total RSS from baseline was more significant in the high-dose group at 12 (difference 0.87 , $p=0.049$ ) and 24 month (difference 1.23, $p=0.011$ ). The serum TALP level was significantly lower in the high-dose group at 6 months. Pi level, height Z-score change, frequency of dental abscess and ratio of de novo nephrocalcinosis were comparable. A lower incidence of secondary hyperparathyroidism was seen in the high-dose group $(p<0.0001)$.

Conclusion For the first time in this prospective cohort, $40 \mathrm{ng} / \mathrm{kg} / \mathrm{d}$ calcitriol was shown to be the more effective therapy in XLH children than the $20 \mathrm{ng} / \mathrm{kg} / \mathrm{d}$. Moreover, $40 \mathrm{ng} / \mathrm{kg} / \mathrm{d}$ calcitriol was not associated with increasing adverse events.

Trial registration ClinicalTrials.gov Identifier: NCT 03,820,518.
\end{abstract}

Keywords X-linked hypophosphatemia $(\mathrm{XLH}) \cdot$ Calcitriol $\cdot$ Phosphate $\cdot$ Randomized $\cdot$ Prospective study

\section{Introduction}

$\mathrm{X}$-linked hypophosphatemia (XLH) is recognized as the most common genetic cause for the rickets. Loss-of-function of PHEX (phosphate-regulating endopeptidase homologue,

W. Xia

xiaweibo8301@163.com

1 Department of Endocrinology, Key Laboratory of Endocrinology, NHC, State Key Laboratory of Complex Severe and Rare Diseases, Peking Union Medical College Hospital, Chinese Academy of Medical Sciences, Shuaifuyuan No. 1, Dongcheng District, Beijing 100730, China
X-linked) gene causes the elevated levels of Fibroblast growth factor 23 (FGF23) [1, 2]. Most manifestations of XLH are caused by FGF23-induced hypophosphatemia leading to downregulation of sodium-phosphate transporters in the renal distal tubule along with the repression of serum $1,25 \mathrm{OH}_{2} \mathrm{D}$ [3]. The features of XLH is more apparent in the first or second year of life. However, early treatment for the disease is related to the better outcomes [4, 5], and therefore, pharmaceutical therapy in pediatrics patients play a key role. Since the 1980s [6], conventional therapy for XLH have been associated with the multiple daily doses of oral phosphate and one or more daily doses of active vitamin $D$ (calcitriol or alfacalcidol). Although conventional therapy leads to variable improvement in the clinical features of 
rickets, it is also associated with the adverse effects such as nephrocalcinosis and hyperparathyroidism. Hence, standardized drug application along with the close monitoring for adverse events are highly warranted. The published guidelines recommends $20-30 \mathrm{ng} / \mathrm{kg}$ body weight daily dose of calcitriol in XLH children [7, 8]; however,other reported doses of calcitriol vary widely, ranging from 10 to $80 \mathrm{ng} /$ $\mathrm{kg} /$ day. Moreover, there is a lack of randomized controlled trials for assessing the clinical evidence for the efficacy and safety of calcitriol in Chinese XLH patients or any other population. We previously reported that in the largest XLH cohort $(N=261)$, less than $50 \%$ had previous treatment of oral phosphate and calcitriol, out of which $34 \%$ patients had received irregular treatment prior the confirmation of the diagnosis [4]. In addition, the XLH children response to treatment varies greatly among individuals. Therefore, systemic evaluation of the drug therapy is very essential.

Calcitriol promotes the absorption of calcium and phosphorus in the intestines and reabsorption in the renal tubules. It directly participates in the regulation of osteogenesis and osteoclast activity, further improving the skeletal microarchitecture and bone strength in the absence of phosphate in XLH [9]. Therefore, a higher dose of calcitriol may better improve bone rickets and promote growth in children. On contrary, excessive use of calcitriol increases the risk of hypercalcemia, and nephrocalcinosis. In 2018, burosumab (a fully human monoclonal antibody against FGF23) was approved for the treatment of X-linked hypophosphatemia, which showed effective and promising results in children and adults, especially a superior efficacy compared to conventional therapy in phase 2 and 3 trials [10-13]. However, an extended observation period is required prior to the burosumab general use in XLH patients, still, it cannot be used widely owing to its high price. Therefore, conventional therapy remains the primary choice in China's population. Therefore, this prospective, randomized, open-label trial was conducted to observe the effects of higher and lower doses of calcitriol combined with neutral phosphate solution in the early treatment of XLH patients.

\section{Participants and methods}

\section{Trial design and participants}

The study was a single-center, randomized, open-label, prospective trial at the Department of Endocrinology, Peking Union Medical College Hospital (PUMCH). This study recruited patients aged 1-12 years of both sexes. Informed consent was signed by either the parents or their guardians of the participants.

Inclusion criteria: (1) PHEX mutation in the participant or a variant of unknown significance in the patient or a family member with appropriate X-linked dominant inheritance, (2) fasting serum phosphate less than the lower limits of the normal range, and (3) a total Thacher rickets severity score (RSS) of at least 2.0. The RSS score (0-10.0) was based on the X-ray findings of metaphysis at wrist and knee, including metaphyseal lucency, metaphyseal fraying and concavity, and physeal widening. The total score was a sum of the two sites [14, 15].

Exclusion criteria: (1) radiological signs of epiphyseal closure; (2) use of growth hormone within 12 months before the first visit; (3) presence of nephrolithiasis; (4) serum parathyroid hormone level over $200 \mathrm{pg} / \mathrm{ml}$ (3 times over the upper limit, which are at risk for hypercalcemia and hyperparathyroidism when using oral phosphate); (5) planning to receive orthopedic surgery within the next 12 months; and (6) the use of aluminum hydroxide, steroid, acetazolamide, or thiazide drugs within 7 days prior to the first visit.

\section{Procedures}

Eligible participants were randomly assigned in a 1:1 ratio to two groups. The follow-up was done at $0,3,6,12,18$, and 24 months. Electronic medical records along with filled case reports were registered for every patient during their visits. The oral phosphate consisted mixed powder of $\mathrm{Na}_{2} \mathrm{HPO}_{4}$ $(29 \mathrm{~g})$, and $\mathrm{KH}_{2} \mathrm{PO}_{4}(6.4 \mathrm{~g})$ dissolved in a 1-L distilled water, where the total phosphate content was $779 \mathrm{mg} / \mathrm{dl}$. In both the groups, the dose of neutral phosphate was $30 \mathrm{mg} / \mathrm{kg}$ per day, divided into four to five doses. High-dose group: The calcitriol dose was $40 \mathrm{ng} / \mathrm{kg}$ per day, with a minimum dose of $0.25 \mu \mathrm{g} /$ day ( 1 tablet) and a maximum of $1 \mu \mathrm{g} / \mathrm{d}$ (4 tablets). Low-dose group: The dose was $20 \mathrm{ng} / \mathrm{kg}$ per day, with a min of $0.125 \mu \mathrm{g} / \mathrm{d}$ (half tablet) to a maximum of $0.5 \mu \mathrm{g} /$ day (2 tablets). In both the groups, calcitriol could be given one to three times a day. The calcitriol dose was adjusted to $40 \mathrm{ng} /$ $\mathrm{kg}$ /day when the serum ALP or PTH level increased over $30 \%$. Whereas the dose of calcitriol decreased to $20 \mathrm{ng} / \mathrm{kg} /$ day when the serum calcium level was over the upper limit, the 24-h urine calcium level was over $4 \mathrm{mg} / \mathrm{kg}$, and newly developed or worsened nephrocalcinosis appeared during the follow-up visit. Calcium intake was controlled to $500 \mathrm{mg} /$ day for each patient.

The severity of rickets was evaluated by radiography, laboratory examinations, and clinical symptoms. Two physicians assessed the Thacher rickets severity score (RSS) [15] at baseline, 12 and 24 months. Body length or standing height, weight, leg deformities, oral examination (for dental abscess, teeth number, and appearance), vision, and hearing tests were performed during the patient's physical examinations. The body height Z-score was also calculated at each visit using age- and sex-matched growth charts for Chinese children and adolescents (aged 0 to 18 years) [16], while the growth velocity was calculated as $\mathrm{cm} /$ year. Self-reported 
daily diet and complaints of discomfort related to treatment were recorded. Drug adherence was confirmed by the analysis of urine phosphate level at each visit. Fasting laboratory tests were obtained in the morning, $1-1.5 \mathrm{~h}$ after taking the phosphate. Total alkaline phosphatase (TALP), serum phosphate, serum calcium, serum parathyroid hormone (PTH), and 24-h urine calcium were assessed throughout the study. Renal ultrasonography was performed at baseline and 12 and 24 months, and nephrocalcinosis was measured with an ordinal scale ranging from normal (0) to stone formation (4) $[10]$.

Assays: Methods used for assaying the analytes are described in details in the supplementary materials.

\section{Outcomes}

Endpoint assessments were compared inter- and intra-group. The primary efficacy endpoint was the total RSS change from the baseline to the 24th month, whereas the secondary efficacy endpoints included the total RSS change from baseline to the 12th month. In addition, changes from baseline in fasting serum TALP levels (time frame: baseline, 3 , $6,12,18$, and 24 months), fasting serum phosphate levels (baseline, 3, 6, 12, 18, and 24 months), body height Z-score (baseline, 12 and 24 months), and frequency of dental abscess (baseline, 12 and 24 months). Safety assessments evaluated adverse events that occurred at any time during the study. Gastrointestinal adverse reactions, renal ultrasound nephrocalcinosis grades (0-4), fasting serum calcium and PTH levels were also recorded.

\section{Statistical analysis}

The study was designed such that a dose of $40 \mathrm{ng} / \mathrm{kg} / \mathrm{day}$ calcitriol would improve the RSS at 24 months compared to $20 \mathrm{ng} / \mathrm{kg} /$ day calcitriol. Assuming the mean total RSS values of 4.8 and 4.0, respectively, and standard deviations of 1.5 at 24 months, a total sample size of 52 (26 in each group) could achieve $81 \%$ power to detect a difference with a significance level (alpha) of 0.05 using a two-sided, two-sample $t$-test. Patients with at least one post-baseline visit were included in the modified intention-to-treat (mITT) analysis for the secondary efficacy and safety parameters. For the main efficacy parameters, both mITT analysis and pre-protocol (PP) analysis were presented. The primary endpoint was the total RSS change from baseline. Repeated measures ANOVA was used to analyze the group $\times$ time effect. The secondary endpoint assessment was body length/height Z-score; fasting laboratory assays were counted as quantitative data, and differences between groups were analyzed using Student's $t$-test or the Mann-Whitney $U$ test. Within-group differences were analyzed with the paired-samples $t$-test or the Wilcoxon signed-rank test. Categorical data were analyzed using the chi-square test and Fisher's exact test. Pearson correlations were evaluated between the total RSS and alkaline phosphatase activity change from baseline in both groups at 12 and 24 months. Significance was defined as a $p$-value less than 0.05. All statistical analyses were computed using the IBM SPSS statistical program (Chicago, USA) version 26.0.

\section{Results}

\section{Participants and dose adjustment}

A total of 72 children were screened (Fig. 1), of whom 68 were found eligible for participation and then randomly assigned to high-dose $(N=36)$ and low-dose group $(N=32)$. Patients were either newly diagnosed, or were recruited from another cohort in our clinic [4]. Three patients did not finish at least one post-baseline visit (high-dose group $(n=1)$ and low-dose group $(n=2)$ ); therefore, their data were not included in any analysis. In addition, 5 patients underwent dose adjustment during the study (Supplementary Table S1). Overall, $53(81.5 \%)$ participants completed the 24 months of the treatment. However, 12 (18.4\%) patients did not strictly comply with the protocol follow-up. The latest visit points included for statistical analysis of these patients were 12 months (one in each group), 17 months (1 in the high-dose group), 18 months (1 in the high-dose group), 19 months ( 2 in the low-dose group, and 1 in the high-dose group), 20 months ( 2 in the low-dose group and 1 in the high-dose group), and 21 months (1 in each group) (Fig. 1). The distance from the hospital was the fundamental reason for the patients who did not follow the schedule.

The calcitriol dose calculated with the body weight was significantly higher in the high-dose group at baseline $(p<0.0001), 12$ months $(p<0.0001)$, and 24 months $(p<0.0001)$ than in the low-dose group (Supplementary Table S2). The radio of calcitriol for three times a day from the beginning of the study was $9.2 \%(6 / 65)$. The baseline clinical characteristics and demographics of the two groups were found to be comparable (Table 1). Children had an average age of $6.12 \pm 2.83$ years at the time of enrolment, with $53.8 \%$ girls' population. Overall, $87.6 \%$ of the patients had received conventional treatment for a mean of 2.25 years before starting the study, while eight patients did not receive any drug therapy before the enrolment. The sex- and agespecific Z-score for height was $-2.06 \pm 1.05$, which indicated short stature in XLH. In total, patients had active rickets at growth plate sites (total RSS score $>2$ ) with an average score of 5.13, where one of the patients scored as high as 10 . Significant symptoms related to rickets included weakness (58.5\%) and bone pain (58.5\%). Overall, 25.5\% of patients reported occasional abscesses, $27.5 \%$ reported frequent tooth abscesses, while $47.1 \%$ of patients did not 
Fig. 1 Study profile. Of the 4 patients who did not meet the inclusion criteria, one patient was finally diagnosed with Fanconi syndrome. One patient carries a disease-causing mutation of ENPP1 gene and is negative for PHEX mutation. One patient was ineligible due to a PTH level greater than $200 \mathrm{pg} / \mathrm{ml}$. One patient has a radiographic feature of epiphyseal closure

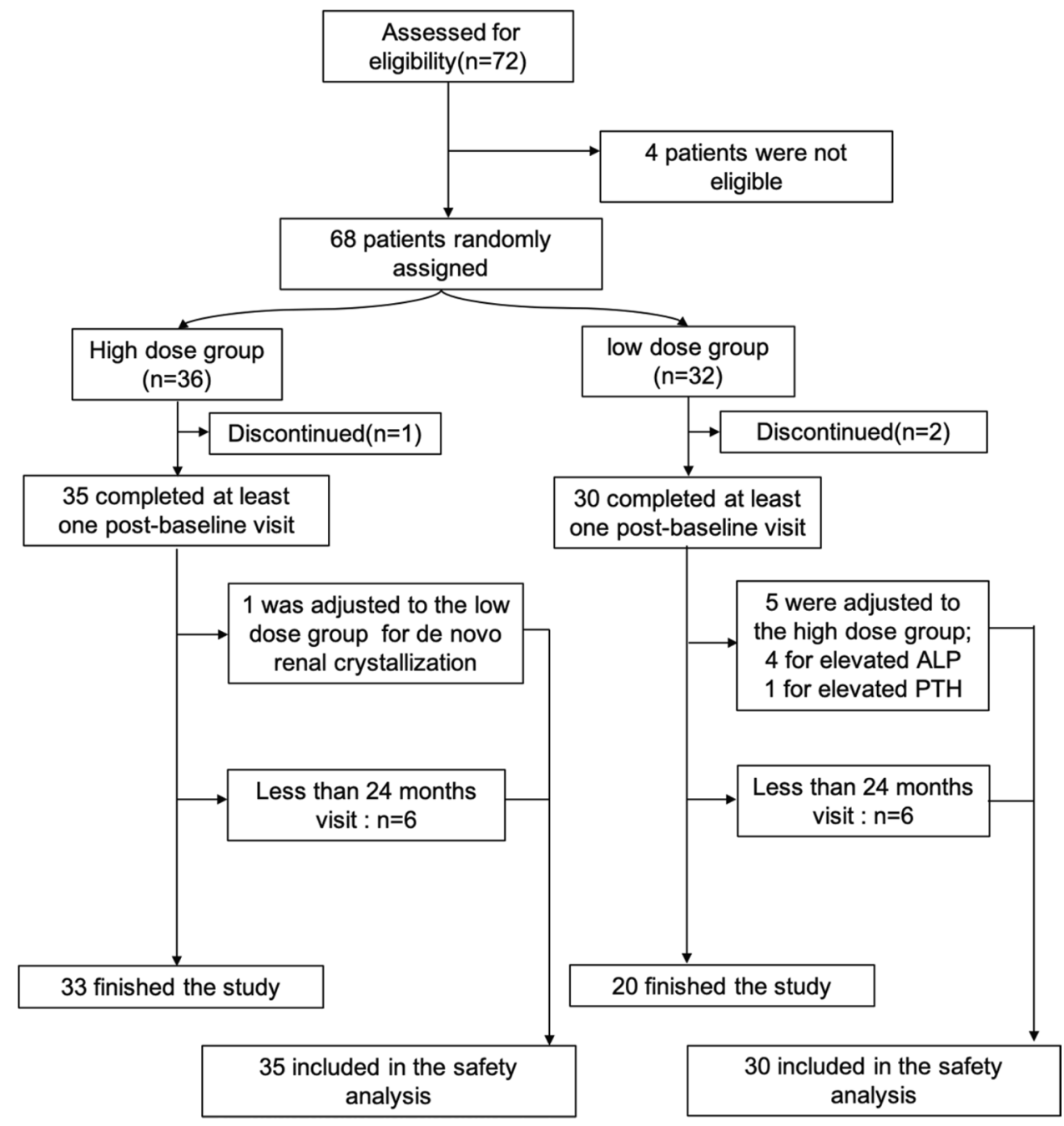

experience any abscesses. At baseline, the serum alkaline phosphatase concentration was $537.9 \pm 127.6 \mathrm{U} / \mathrm{L}$; the median serum phosphate concentration was $0.83 \mathrm{mmol} / \mathrm{L}$, the serum $1,25(\mathrm{OH})_{2} \mathrm{D}$ concentration was $39.1 \pm 23.2 \mathrm{pg} /$ $\mathrm{mL}$, and the serum PTH level in $23.8 \%(15 / 63)$ of patients was above the normal range $(12-68 \mathrm{pg} / \mathrm{mL})$.

\section{Efficacy}

At 12 months, in the low-dose group, the total RSS improved from baseline in $35.3 \%(6 / 17)$ of patients. In the high-dose group, total RSS improvement seen in $57.1 \%(16 / 28)$ of patients. At 24 months, the total RSS improvement from baseline was observed in $61.1 \%(11 / 18)$ patients, where the ratio was $80.0 \%(20 / 25)$ of patients in the high-dose group. For the primary efficacy endpoint, the total RSS in the highdose group at 24 months was decreased markedly from baseline compared with the low-dose group [mean change from baseline - 1.82 (SE 0.32) versus - 0.58 (SE 0.33), respectively; difference $1.23, p=0.011$ (Fig. 2A)]. Similar results were observed for the mITT analysis (Supplementary Table S3). For total RSS change from baseline to 12 months, the difference in the high-dose group was still more apparent [mean change from baseline - 1.03(SE 0.28) vs. $-0 \cdot 17$ (SE $0 \cdot 31$ ), respectively; difference $0.87, p=0.049$, Fig. 2A]. Total RSS significantly decreased at 12 and 24 months compared with baseline score $(4.77 \pm 1.71$ and $4.53 \pm 1.64$ vs. $5.91 \pm 1.84$ at baseline, $p=0.001, p<0.0001$ ) (Fig. 2B), while no such differences were observed in the low-dose group. A mild correlation was found between change in total RSS with alkaline phosphatase activity from baseline to 24 months $(r=0.35, p=0.024)$ (Fig. 2D). A negative correlation was observed between the changes in RSS and ALP for the low-dose group.

As early as 6 months, the alkaline phosphatase activity was significantly lowered in the high-dose group than the low-dose group (Fig. 3A). The mean changes in ALP level from baseline were $1.47 \%, 4.2 \%, 14.5 \%, 16.8 \%$, and $17.0 \%$ in the low-dose group at $3,6,12,18$, and 24 months, respectively, while the mean changes in the high-dose group 
Table 1 Baseline demographics

\begin{tabular}{|c|c|c|c|c|}
\hline & Total $N=65$ & Low-dose group $N=30$ & High-dose group $N=35$ & $P$ value $^{\mathrm{f}}$ \\
\hline Female, N (\%) & $35(53.8)$ & $21(70)$ & $14(40)$ & \\
\hline Age when enrolled & $6.12 \pm 2.83$ & $6.58 \pm 2.69$ & $5.72 \pm 2.92$ & 0.459 \\
\hline \multicolumn{5}{|l|}{ Height } \\
\hline $\mathrm{cm}$ & $106.52 \pm 17.02$ & $109.8 \pm 15.92$ & $103.71 \pm 17.65$ & 0.453 \\
\hline $\operatorname{SDS}^{\mathrm{a}}$ & $-2.065 \pm 1.05$ & $-1.98 \pm 1.12$ & $-2.02 \pm 0.96$ & 0.453 \\
\hline \multicolumn{5}{|l|}{ Weight } \\
\hline $\mathrm{Kg}$ & $19(15,24.25)$ & $20.1(17.75,27.25)$ & $18.0(14.0,23.0)$ & 0.115 \\
\hline $\operatorname{SDS}^{\mathrm{a}}$ & $-0.604(-0.907,0.459)$ & $-0.44(-0.87,0.72)$ & $-0.62(-1.44,0.21)$ & 0.498 \\
\hline \multicolumn{5}{|l|}{ Treatment before enrolled } \\
\hline $\mathrm{N}(\%)$ & $57(87.6)$ & $28(93.3)$ & $29(82.8)$ & \\
\hline Years & $2.25(0.979,3.749)$ & $2.77(1.60,4.20)$ & $1.17(0.58,3.39)$ & 0.161 \\
\hline Previous orthopedic surgery & $6(9 \%)$ & $1(3 \%)$ & $5(14.2 \%)$ & \\
\hline \multicolumn{5}{|l|}{ Symptoms and signs, N (\%) } \\
\hline weakness & $38(58.5 \%)$ & $16(53.30 \%)$ & $22(62.9 \%)$ & \\
\hline bone pain & $38(58.5 \%)$ & $19(63.30 \%)$ & $19(54.3 \%)$ & \\
\hline leg deformity ${ }^{\mathrm{b}}$ & $53(81.5 \%)$ & $22(76.7 \%)$ & $30(85.7 \%)$ & \\
\hline \multicolumn{5}{|l|}{ periodontal abscess $^{c}$} \\
\hline negative & $24(47.1 \%)$ & $11(55 \%)$ & $13(41.9 \%)$ & \\
\hline occasional & $13(25.5 \%)$ & $4(20 \%)$ & $9(29 \%)$ & \\
\hline frequent & $14(27.5 \%)$ & $5(25 \%)$ & $9(29 \%)$ & \\
\hline $\mathrm{Ca}(\mathrm{mmol} / \mathrm{L})^{\mathrm{d}}$ & $2.42 \pm 0.08$ & $2.44 \pm 0.076$ & $2.4 \pm 0.088$ & 0.478 \\
\hline $\mathrm{Pi}(\mathrm{mmol} / \mathrm{L})^{\mathrm{d}}$ & $0.83(0.77,0.92)$ & $0.835(0.77,0.95)$ & $0.82(0.77,0.86)$ & 0.469 \\
\hline $\operatorname{TALP}(\mathrm{U} / \mathrm{L})^{\mathrm{d}}$ & $537.9 \pm 127.6$ & $566.5 \pm 118.14$ & $512.8 \pm 132.0$ & 0.093 \\
\hline $\mathrm{Cr}(\mu \mathrm{mol} / \mathrm{L})^{\mathrm{d}}$ & $30.02 \pm 7.39$ & $30.55 \pm 7.56$ & $29.56 \pm 7.33$ & 0.885 \\
\hline $\mathrm{T} 25 \mathrm{OHD}(\mathrm{ng} / \mathrm{ml})^{\mathrm{d}}$ & $25.05(21,36.37)$ & $25.7(21.35,35.25)$ & $23.8(20.65,36.45)$ & 0.71 \\
\hline $1,25(\mathrm{OH}) 2 \mathrm{D} 3(\mathrm{pg} / \mathrm{ml}) \mathrm{d}$ & $39.1 \pm 23.2$ & $43.51 \pm 25.21$ & $36.30 \pm 22.28$ & 0.452 \\
\hline PTH $(\mathrm{pg} / \mathrm{ml})^{\mathrm{d}}$ & $38.5(28.8,62.9)$ & $37.95(26.27,52.45)$ & $41.80(29.40,86.55)$ & 0.325 \\
\hline $24 \mathrm{~h}-\mathrm{UCa}(\mathrm{mmol} / \mathrm{L})^{\mathrm{d}}$ & $0.74(0.327,1.525)$ & $0.67(0.35,1.24)$ & $0.9(0.3,2)$ & 0.434 \\
\hline \multirow[t]{2}{*}{ Thacher rickets severity score ${ }^{\mathrm{e}}$} & $5.13(1.75)$ & $4.91(1.54)$ & $5.32(1.91)$ & 0.362 \\
\hline & $N=60$ & $N=28$ & $N=32$ & \\
\hline
\end{tabular}

* Data are mean $\pm \mathrm{SD}$, median (IQR) or $\mathrm{N}(\%)$

Ca calcium; Pi phosphate; TALP total alkaline phosphatase; Cr creatinine; T25OHD total 25-hydroxyvitamin D; 1, 25(OH)2D3 1,25-Dihydroxyvitamin D; PTH parathyroid hormone; U-Ca urinary calcium

${ }^{a}$ Standing height $\mathrm{Z}$-score and body weight $\mathrm{Z}$-score adjusted for sex and age

${ }^{\mathrm{b}}$ Knee varus and valgus

${ }^{\mathrm{c}}$ Self-reported frequency of tooth abscess

${ }^{\mathrm{d}}$ Normal range in PUMCH: calcium 2.13-2.7mmol/L; phosphate in age and sex groups:1-5 1.45-2.1mmol/L, 6- 12 1.2-1.9mmol/L;upper limit of ALP in age and sex groups: males- 1 to 10 years 335U/L, 10 to 13 years 417U/L; females- 1 to 10 years 335U/L, 10-13 years 417U/L; S-Cr 18-69 $\mu \mathrm{mol} / \mathrm{L}$; T-25OHD 8-50ng/ml;1,25(OH)2D3 19.6-54.3 pg/mL; PTH 12-68pg/mL

${ }^{\mathrm{e}}$ Total Thacher rickets severity score

${ }^{\mathrm{f}}$ difference between the two groups

were $-4.1 \%,-4.5 \%, 6.3 \%, 2.4 \%$, and $4.5 \%$, respectively. The differences between the two groups were not statistically significant.

Phosphate level was significantly increased from baseline to 24 months in both groups [(in the high-dose group, $0.93(0.77,1.24) \mathrm{mmol} / \mathrm{L}$ at month 24 versus $0.83(0.56$, 1.44) $\mathrm{mmol} / \mathrm{L}$ at baseline, $p=0.001)$ ]; [in the low-dose group, $0.83(0.75,1.28) \mathrm{mmol} / \mathrm{L}$ at month 24 versus 0.83 $(0.59,1.24) \mathrm{mmol} / \mathrm{L}$ at baseline, $p=0.013$ ] (Fig. 3B).In the high-dose group, phosphate level also increased from baseline to 12 month $[0.88(0.69,1.24) \mathrm{mmol} / \mathrm{L}, p<0.01]$ and 18 months $[0.88(0.65,1.3) \mathrm{mmol} / \mathrm{L}, p<0.01]$. No difference was observed in the fasting phosphate levels from baseline at any time of the study for the two groups. We compared the 
A

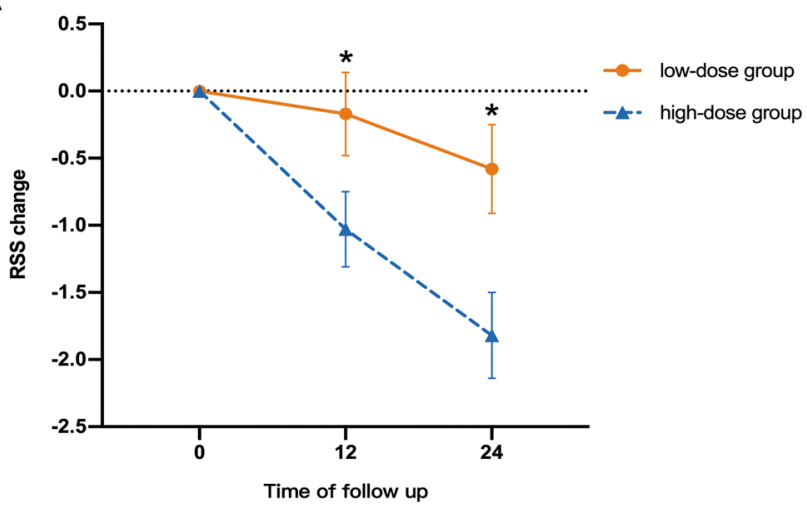

B

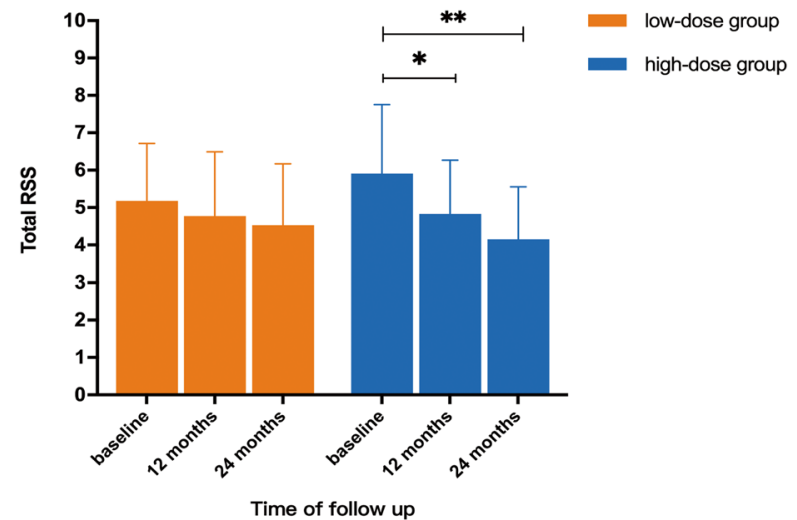

D

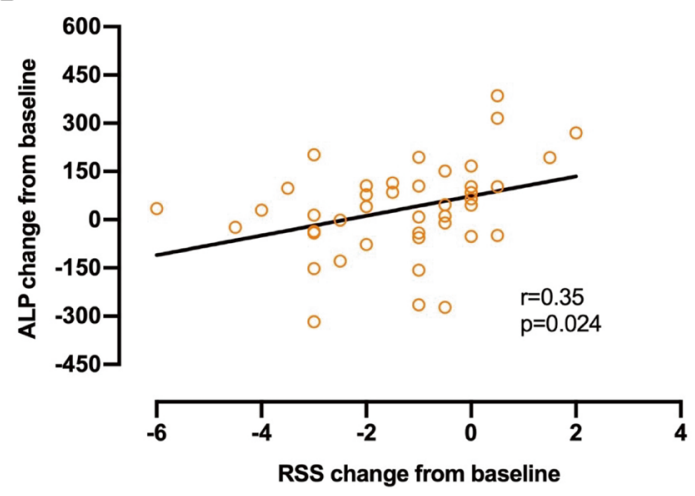

Fig. 2 A, B, C, D Improvement in rickets severity score. Data in panels of $\mathbf{A}$ and $\mathbf{B}$ are shown in mean \pm standard deviation. $\mathbf{A}$ Mean change of total RSS change from baseline of the two groups at months 12 and $24 ;{ }^{*} p<0.05$ based on the comparison between treatment groups using the repeated measurement ANOVA model. B RSS change with treatment within groups. ${ }^{\#} p<0.01,{ }^{\# \# \# p<0.0001}$ based on the comparison from baseline in the mean difference using

serum phosphate level/the upper limit of the age group both at baseline and month 24. At month 24, 68.4\% (13/19) of patients in the low-dose group had phosphate values above the normal range; the ratio in the high-dose group was $81.8 \%$ (27/33). The difference between the two groups was significant $(p=0.003)$.
C RSS change in a 3.26-year gril in the high dose group
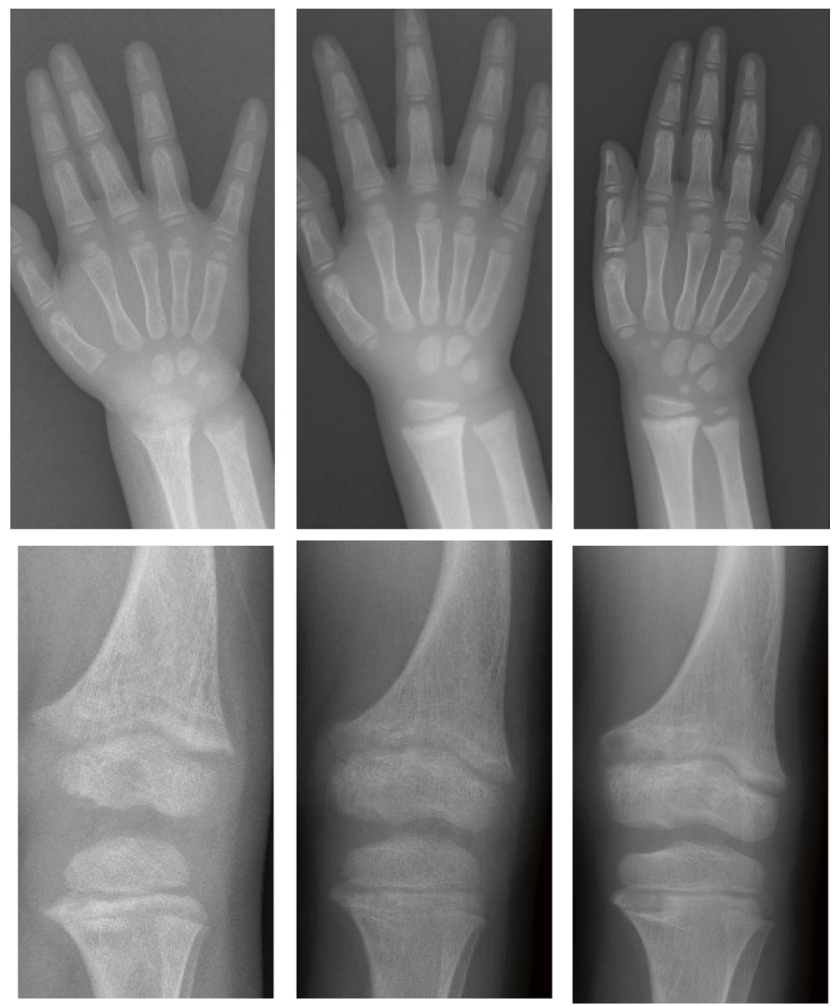

baseline

month 12

month 24

wrist 4

knee 2

total 6

wrist 2.5

wrist 2

knee 1

knee 1

total 3.5

total 3

the paired-sample $T$ test at months 12 and 24. Radiographs in figure panel $\mathbf{C}$ show rickets score improvement in a 3-year-old girl in the high-dose group from baseline to month 24 . From total Thacher severity score of 6 (wrist 4 , knee 2) to 3 (wrist 2, knee 1) at month 24. D A mild correlation between RSS and ALP change from baseline to month 24 in the high-dose group

At baseline, the mean height $\mathrm{Z}$-score was $-2.14 \pm 1.05$ in the low-dose group and $-2.17 \pm 1.03$ in the highdose group. At month 12, the mean size Z-score was $-2.00 \pm 0.97$ in the low-dose group and $-2.11 \pm 1.06$ in the high-dose group. At month 24, the mean height $\mathrm{Z}$-score was $-1.92 \pm 0.94$ in the low-dose group and 

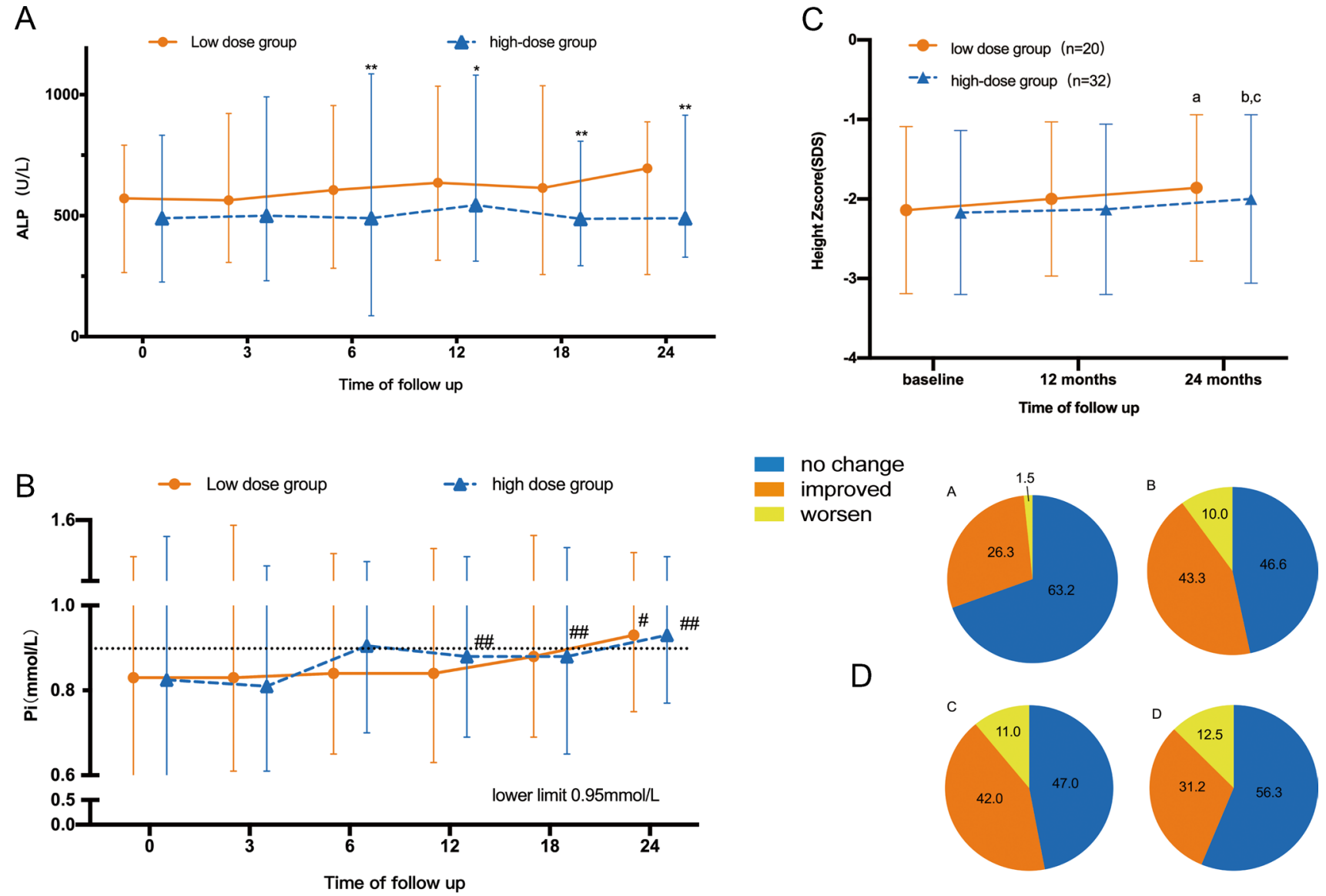

Fig. 3 Secondary efficacy endpoints (A, B, C, D). Panels of A and $\mathbf{B}$ are shown in median (min, max). Data in panel $\mathbf{C}$ is shown in mean \pm standard deviation. Panel $\mathbf{D}$ is shown in percentage. ${ }^{*} p<0.05,{ }^{* *} p$ $<0.01$ based on the on the comparison between treatment groups using Wilcoxon signed-rank test in (A). ${ }^{\#} p<0.05,{ }^{\# \#} p<0.01$ based on the comparison from baseline in the mean difference using MannWhitney $U$ test in (B). a, b, and c were paired-sample $T$ test in $(\mathbf{C})$. a ( $p=0.021$ ) was the comparison of height $Z$ score of month 24 in the low-dose group from baseline. $\mathrm{b}(p=0.002)$ and $\mathrm{c}(p=0.017)$ the com-

$-1.96 \pm 1.04$ in the high-dose group. Thus, the mean height $\mathrm{Z}$-score at month 24 compared to baseline was significantly elevated in both groups $(p=0.021$; $p=0.002$ ), and the mean score was considerably higher in the high-dose group $(p=0.017)$ alone than the high Z-score group at 12 months (Fig. 3C). The mean height $\mathrm{Z}$-score at 12 months compared to the baseline was not significantly different in any group.

A total of 51 patients (low-dose group $(N=20)$ and highdose group $(N=31))$ had baseline information on dental abscesses (Fig. 3D). By the end of 24 months, 17/51 (33.3\%) patients did not experience at least one dental abscess (8/19 in the low-dose group and 9/32 in the high-dose group). At 12 months, ratios of $15.8 \%$ (3/19), 26.3\% (5/19), and $10.5 \%(2 / 19)$ in the low-dose group reported no change or a decreased or increased frequency of tooth abscess, while parison of height $Z$ score of month 24 in the high-dose group from baseline and month 12 . Tooth abscess frequency change from baseline in the low-dose group at month $12(\mathbf{A}, \mathbf{B}, \mathbf{C}, \mathbf{D})$ and at month 24 (B, C, D). 19 patients in the low-dose group and 30 in the highdose group tooth abscess frequency change from baseline in the highdose group at 12 months $(\mathbf{B}, \mathbf{C}, \mathbf{D})$ and at 24 months (D). Ratio of patients without tooth abscess during the whole study was counted as no change group

Table 2: Adverse events

\begin{tabular}{lll}
\hline Assessment & Group \\
\cline { 2 - 3 } Change in nephrocalcinosis score & Low dose group & $\begin{array}{l}\text { High } \\
\text { dose } \\
\text { group }\end{array}$ \\
\hline Increased by 1 point & $1(6)$ & $2(9)$ \\
Increased by $>1$ point & 0 & 0 \\
Decreased by 1 point & 0 & $2(9)$ \\
Decreased by $>1$ point & 0 & 0 \\
Secondary hyperparathyroidism & $9(30)$ & $(20)$ \\
\hline
\end{tabular}

*Data is in number(percentage). Adverse events are recorded at any time during the study. PTH level over $68 \mathrm{pg} / \mathrm{ml}$ at any post-baseline assessment is counted as secondary hyperparathyroidism. Other symptoms are self-reported. 


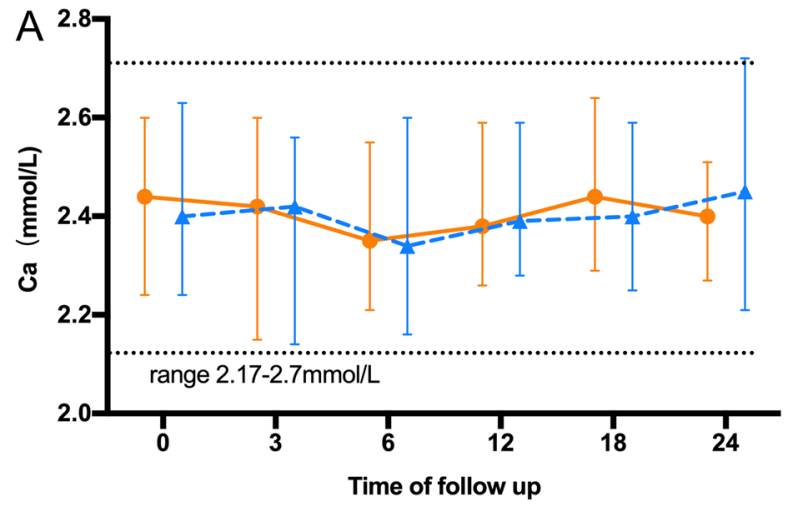

B
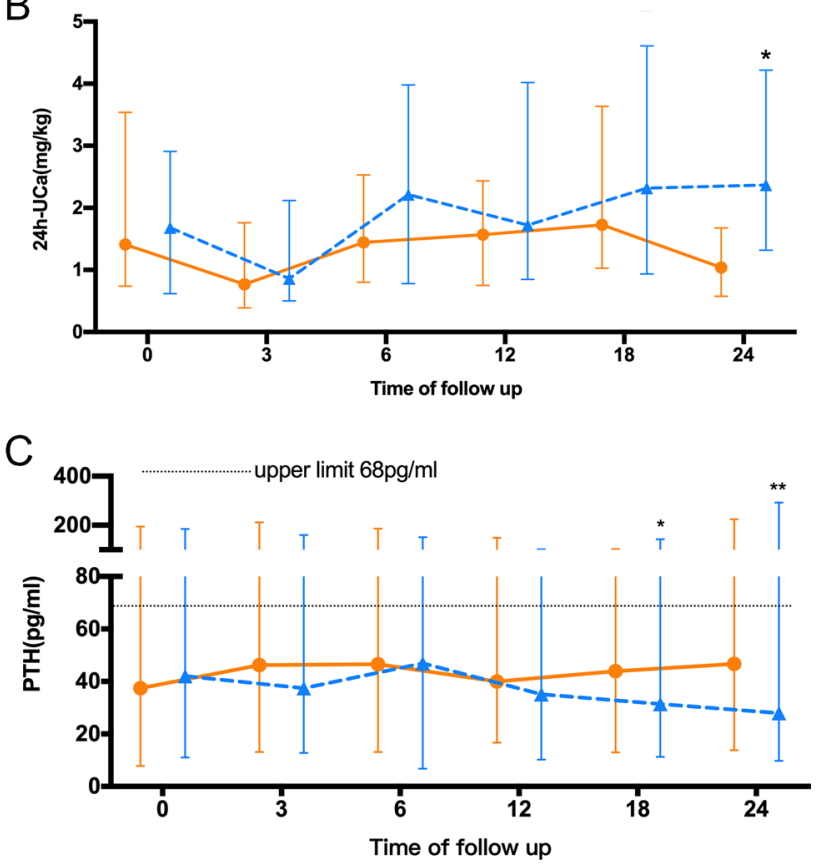

Fig. 4 Serum calcium and urine calcium level (A, B, C). Panels of $\mathbf{A}$ and $\mathbf{B}$ are shown in median $(\min , \max )$. No difference in serum calcium was observed (A). ${ }^{*} p<0.05$ based on the on the comparison between treatment groups using Wilcoxon signed-rank test in (B). 24h-UCa was higher in the high-dose group at month 24. C PTH level was lower in the high-dose group since month 18

the ratios in the high-dose group were $13.3 \%$ (4/30), $43.3 \%$ $(13 / 30)$, and $10.0 \%(3 / 30)$, respectively. At 24 months, the ratios were $10 \%(2 / 20), 40 \%(8 / 20)$, and $10 \%(2 / 20)$ in the low-dose group and $25.8 \%(8 / 31), 32.2 \%$ (10/31), and $12.9 \%(4 / 31)$ in the high-dose group. There were no significant differences observed between the two groups in any assessment.

\section{Safety}

Hyperparathyroidism related to treatment was observed in both groups (Table 2). A total of $16(26.1 \%)$ patients, 9 (30\%) patients in the low-dose group, and 7 (20\%) patients in the high-dose group developed de novo hyperparathyroidism during the study period. The ratio of secondary hyperparathyroidism in the low-dose group was significantly higher than that in the high-dose group $(p<0.0001)$. PTH level was much lower in the high-dose group at 18 months (Fig. 4C). No hypercalcemia and hyperparathyroidism occurred in either of the group. Changes in serum calcium were minimal in both the groups, with no significant difference found between the groups (Fig. 4A) except for one patient in the high-dose group. In this patient, the serum calcium level was $2.7 \mathrm{mmol} / \mathrm{L}$ at month 24 , while the PTH level was in the normal range $(19.3 \mathrm{pg} / \mathrm{ml})$. At month 24 , the urinary calcium $(\mathrm{mg} / \mathrm{kg} / 24 \mathrm{~h})$ of the high-dose group was $2.36(1.32,4.22)$, higher than that in the low-dose group $(\mathrm{mg} / \mathrm{kg} / 24 \mathrm{~h}): 1.04$ $(0.58,1.68),(p=0.016)$. There was no statistical difference in $24 \mathrm{~h}$ urinary calcium levels between the two groups at any other visit (Fig. 4B).

Baseline renal ultrasound was available for the 37 (56.9\%) patients. A total of 70.2\% (26/37) patients showed no sign of nephrocalcinosis in renal ultrasound (grade 0 ), while $5.4 \%$ (2/37), 8.1\% (3/37), 8.1\% (3/37), and $8.1 \%$ (3/37) patients had grades $1-3$ nephrocalcinosis. A total of 3 patients had a high grade of nephrocalcinosis, and 2 patients in the high-dose group showed decreased grade of nephrocalcinosis. The percentages of newly developed nephrocalcinosis between the two groups had no significant differences (Table 2). Diarrhea or any other gastrointestinal discomfort was not reported in either of the group throughout the study.

\section{Discussion}

Although XLH has been recognized for decades, due to the disease's rarity and the diversity of clinical manifestations, diagnosis is often delayed, and therefore treatment can be challenging [8]. This present study conducted the first prospective study on large cohort to explore the efficacy as well as the safety of high-/low-dose calcitriol combined with neutral phosphorus in treating children with XLH.

At baseline, patients in these two groups shared same severity of rickets and other clinical features. Although more girls were included in the low-dose group than in the high-dose group (70\% vs. $40 \%$ ), patients were enrolled and assigned randomly with no sexual preference. All patients reported good drug adherence during the entire study. Notably, at the time of enrolment, participant's baseline total RSS $(5.13 \pm 1.75)$ was much higher than the previous study, although a majority $(87.6 \%)$ of patients were previously treated. In a prior study, mean total RSS scores of 1.8 and 3.0 were reported by Carpenter et al. and Imel, E. A. et al., respectively $[10,13]$. As treatment initiation at early infancy $(<1.0$ year) results in improved outcomes 
in patients with XLH [5], we assumed that the relatively late diagnosis and treatment in our cohort (median age of 2.19), might have contributed to the relatively severe disease burden.

The ALP levels are reported marker reflecting the bone mineralization status in rickets. A previous study has reported decreased ALP concentrations to the upper limit of normal in one year for the conventional therapy [17]. We observed that the ALP level in the high-dose group was significantly lower than the low-dose group after 6 months of treatment. In the 24th month, the average ALP level in the high-dose group almost comparable to the normal range. In line with Thacher et al., who reported an improvement in RSS correlated with decreased serum ALP $(r=0.47)$ in 5- to 12-year-old patients [15], our study has also observed a mild correlation at 24 months. However, the ALP activity had an increase in the low-dose group, which was not found in the previous study [13]. This might be due to the insufficient efficacy of low-dose calcitriol for improving the rickets severity. Besides, the control of the ALP level in the high-dose group were far from satisfactory, which also might be a limitation of the conventional therapy.

Consistent with the previous study, improvement of height $\mathrm{Z}$-score was observed at 24 months in both groups (Fig. 3C) [18, 19], whereas no difference was observed between the groups. Low dentin mineralization, abnormal pulp, and periodontitis are common the abnormalities associated with the X-linked hypophosphatemia, though severity might vary among the patients. Previous reports have suggested that conventional therapy can improve the dentin mineralization as well as decreases the risk of dental abscesses and severe periodontal disease over the years [20, 21]. In the present study, the frequency of dental abscesses was measured by three degrees. Interestingly, the ratio of aggravated dental abscesses was higher in the high-dose group at 24 month, though the ratio could not be correlated with the improvement of rickets or the correction of the serum phosphate. As dental development and abnormalities acquired in utero may alter the response of dental anomalies in children, more extended observation is required for the proper explanation of such incidences.

In safety events, conventional treatment with phosphate supplementation and active vitamin D might increase calcinuria risk and promote nephrocalcinosis in $60 \%$ of children with XLH [22]. In our study, the percentage was much low, where both treatment groups shared a similar ratio of de novo nephrocalcinosis. Additionally, urinary calcium at $24 \mathrm{~h}$ was also not different between the two groups. Hyperparathyroidism is another commonly reported adverse event observed in adult and pediatric XLH patients during conventional treatment [23, 24]. Recently, a retrospective study reported an $81.8 \%$ secondary hyperparathyroidism ratio in 44 XLH children [25].
In addition, we previously reported elevated serum PTH levels in $59.2 \%$ of XLH patients $(N=61 / 103)$ [4]. In the current study, some patients in both groups developed secondary hyperparathyroidism; however, serum PTH levels varied among these patients. Six patients (4 in the high-dose group) had continuously excessive PTH levels, and the others had intermittently elevated PTH levels. No patients had hypercalcemia and hyperparathyroidism. It could be specifically related to oral phosphate, since a recent study demonstrated that phosphate directly regulates PTH secretion through calcium-sensing receptors on parathyroid cells [26]. To understand this further, we compared PTH value in patients who have or have not demonstrated increased serum phosphate values (Supplementary Table S4). Although PTH levels were higher, no significance observed in patients with increasing $\mathrm{Pi}$ value. We previously published that higher PTH level were more persistent in the hypophosphatemic patients than the control group after the oral phosphate loading test $[27,28]$. Thus, it was reasonable to correlate the occurrence of hyperparathyroidism with oral phosphate usage, as we presumed that in hypophosphatemic patients, parathyroid might react more sensitively to the phosphate load. Nevertheless, hyperparathyroidism prevalence can be reversed by a higher compensation of $1,25(\mathrm{OH})_{2} \mathrm{D}$.

The present study had several limitations. First, the trial was open-label; thus, the adjustment upon the occurrence adverse drug reactions was done. It was done to ensure the therapeutic effect and avoid further aggravated adverse reactions. Moreover, to make the results reliable, two different physicians independently assessed the rickets severity score, and a central laboratory assessed pharmacodynamic measures. Another limitation was that we did not compare the FGF23 level before and after the treatment, which might be one of the reasons for increasing level of the serum FGF23, which may further diminish therapeutic effect or contribute to the complications of therapy. It is well established that calcitriol increases osteoblastic production and the serum levels of FGF23, even in healthy individuals [29-31]. However, in our previous study, which included 261 Chinese XLH patients, serum iFGF23 levels did not correlate with serum phosphate/upper limit ratio, age at which disease symptom developed, height SDS, or RSS [4]. This result could provide us with evidence that might be helpful to interpret the severity of the disease from the level of FGF23. Further studies are expected to validate and interpret these findings. As we used fixed dose of phosphate in both groups, more systematic evaluation on both conventional drugs could not be performed. Further studies are required on varied drug doses to provide more robust evidence. 


\section{Conclusion}

For the first time, the current study compared the different doses of calcitriol in a relatively large cohort of Chinese XLH patients. The higher doses of calcitriol ( $40 \mathrm{ng} / \mathrm{kg} /$ day) in the treatment of children with XLH tend to have better efficacy within the acceptable safety. This study provides evidence-based medical evidence for the optimal therapeutic dose of calcitriol for treating children with XLH. Moreover, the study results can be used as an essential theoretical support for clinical guidelines and consensus in this field. Further studies are required on the larger sample size and extended follow-up time for validating the findings of this study.

Supplementary Information The online version contains supplementary material available at https://doi.org/10.1007/s00198-021-06221-w.

Acknowledgements We appreciate our patients and their families for their participating in this study.

Author contributions Study design: WBX. Study conduct: CXJ, CZ, ZZ, LJX and WBX. Data collection: CXJ, CZ, ZZ, LJX,BW, YC, RJ, YJ, OW, ML, XXP, XWM and WX. Data analysis: CXJZ and WBX. Data interpretation: CXJ and WBX. Drafting manuscript: CXJ and WBX. Revising manuscript content: CXJ and WBX. Approving final version of manuscript: ALL.

Funding This work was supported by the National Key R\&D Program of China (2018YFA 0800801), CAMS Innovation Fund for Medical Sciences (No.2016-I2M-3-003), and National Natural Science Fund (No. 81970757).

The present study was approved by the Ethics Committee of Peking Union Medical College Hospital(Zs-1116). Written informed content was obtained from all subjects, and this study was carried out according to the principles defined in the Declaration of Helsinki.

\section{Declarations}

\section{Conflicts of interest None.}

Open Access This article is licensed under a Creative Commons Attribution-NonCommercial 4.0 International License, which permits any non-commercial use, sharing, adaptation, distribution and reproduction in any medium or format, as long as you give appropriate credit to the original author(s) and the source, provide a link to the Creative Commons licence, and indicate if changes were made. The images or other third party material in this article are included in the article's Creative Commons licence, unless indicated otherwise in a credit line to the material. If material is not included in the article's Creative Commons licence and your intended use is not permitted by statutory regulation or exceeds the permitted use, you will need to obtain permission directly from the copyright holder. To view a copy of this licence, visit http://creativecommons.org/licenses/by-nc/4.0/.

\section{References}

1. A gene (PEX) with homologies to endopeptidases is mutated in patients with X-linked hypophosphatemic rickets (1995) The HYP Consortium Nat Genet 11(2):130-6

2. Bergwitz C, Juppner H (2010) Regulation of phosphate homeostasis by PTH, vitamin D, and FGF23. Annu Rev Med 61:91-104

3. Beck-Nielsen SS, Mughal Z, Haffner D et al (2019) FGF23 and its role in X-linked hypophosphatemia-related morbidity. Orphanet J Rare Dis 14(1):58

4. Zhang C, Zhao Z, Sun Y et al (2019) Clinical and genetic analysis in a large Chinese cohort of patients with X-linked hypophosphatemia. Bone 121(212): 20

5. Makitie O, Doria A, Kooh SW et al (2003) Early treatment improves growth and biochemical and radiographic outcome in X-linked hypophosphatemic rickets. J Clin Endocrinol Metab 88(8):3591-3597

6. Glorieux FH, Marie PJ, Pettifor JM et al (1980) Bone response to phosphate salts, ergocalciferol, and calcitriol in hypophosphatemic vitamin D-resistant rickets. N Engl J Med 303(18):1023-1031

7. Carpenter TO, Imel EA, Holm IA et al (2011) A clinician's guide to X-linked hypophosphatemia. J Bone Miner Res 26(7):1381-1388

8. Haffner D, Emma F, Eastwood DM et al (2019) Clinical practice recommendations for the diagnosis and management of X-linked hypophosphataemia. Nat Rev Nephrol 15(7):435-455

9. Liu ES, Martins JS, Raimann A et al (2016) 1,25-Dihydroxyvitamin D alone improves skeletal growth, microarchitecture, and strength in a murine model of XLH, despite enhanced FGF23 expression. J Bone Miner Res 31(5):929-939

10. Carpenter TO, Whyte MP, Imel EA et al (2018) Burosumab therapy in children with X-linked hypophosphatemia. N Engl J Med 378(21):1987-1998

11. Insogna KL, Briot K, Imel EA et al (2018) A randomized, doubleblind, placebo-controlled, phase 3 Trial evaluating the efficacy of burosumab, an anti-FGF23 antibody, in adults with X-linked hypophosphatemia: week 24 primary analysis. J Bone Miner Res 33(8):1383-1393

12. Whyte MP, Carpenter TO, Gottesman GS et al (2019) Efficacy and safety of burosumab in children aged 1-4 years with X-linked hypophosphataemia: a multicentre, open-label, phase 2 trial. Lancet Diabetes Endocrinol 7(3):189-199

13. Imel EA, Glorieux FH, Whyte MP et al (2019) Burosumab versus conventional therapy in children with X-linked hypophosphataemia: a randomised, active-controlled, open-label, phase 3 trial. Lancet 393(10189):2416-2427

14. Thacher TD, Fischer PR, Pettifor JM et al (2000) Radiographic scoring method for the assessment of the severity of nutritional rickets. J Trop Pediatr 46(3):132-139

15. Thacher TD, Pettifor JM, Tebben PJ et al (2019) Rickets severity predicts clinical outcomes in children with X-linked hypophosphatemia: utility of the radiographic rickets severity score. Bone 122(76):81

16. LI H, Cheng YJ, Zong XN, Zhang YQ (2009) Height and weight standardized growth charts for Chinese children and adolescents aged 0 to 18 years. Zhonghua Er Ke Za Zhi 47:487-492

17. Linglart A, Biosse-Duplan M, Briot K et al (2014) Therapeutic management of hypophosphatemic rickets from infancy to adulthood. Endocr Connect 3(1):R13-30

18. Balsan S, Tieder M (1990) Linear growth in patients with hypophosphatemic vitamin D-resistant rickets: influence of treatment regimen and parental height. J Pediatr 116(3):365-371

19. Ariceta G, Langman CB (2007) Growth in X-linked hypophosphatemic rickets. Eur J Pediatr 166(4):303-309

20. Connor J, Olear EA, Insogna KL et al (2015) Conventional therapy in adults with X-linked hypophosphatemia: effects on enthesopathy and dental disease. J Clin Endocrinol Metab 100(10):3625-3632 
21. Biosseduplan M, Coyac BR, Bardet C et al (2017) Phosphate and vitamin D prevent periodontitis in X-linked hypophosphatemia. J Dent Res 96(4):388-95

22. Arango sancho $P$ (2020) Complications of phosphate and vitamin D treatment in X-linked hypophosphataemia. Adv Ther 37(Suppl 2):105-12

23. Su DH, Liao KM, Chang YC et al (2006) Secondary hyperparathyroidism as a palpable intrathyroid parathyroid gland in a patient with hypophosphatemic osteomalacia. J Bone Miner Metab 24(2):114-117

24. McHenry CR, Mostafavi K, Murphy TA (2006) Tertiary hyperparathyroidism attributable to long-term oral phosphate therapy. Endocr Pract 12(3):294-298

25. Delacey S, Liu Z, Broyles A et al (2019) Hyperparathyroidism and parathyroidectomy in X-linked hypophosphatemia patients. Bone 127:386-92

26. Centeno PP, Herberger A, Mun HC et al (2019) Phosphate acts directly on the calcium-sensing receptor to stimulate parathyroid hormone secretion. Nat Commun 10(1):4693

27. Condon JR, Nassim JR, Rutter A (1970) Defective intestinal phosphate absorption in familial and non-familial hypophosphataemia. Br Med J 3(5715):138-141
28. WB. H X X, AL. E. 2009 Acute effects of oral phosphate-salt loading on serum phosphate and parathyroid hormone in hypophosphatemic rickets / osteomalacia. . ZhongHuaGuZhiShuSongYuGUKuangYanJiBing, 2(4): 243-8.

29. Imel EA, Dimeglio LA, Hui SL et al (2010) Treatment of X-linked hypophosphatemia with calcitriol and phosphate increases circulating fibroblast growth factor 23 concentrations. J Clin Endocrinol Metab 95(4):1846-1850

30. Georgiadou E, Marketou H, Trovas G et al (2017) Effect of calcitriol on FGF23 level in healthy adults and its dependence on phosphate level. In Vivo 31(1): 145-150

31. Antoniucci DM, Yamashita T, Portale AA (2006) Dietary phosphorus regulates serum fibroblast growth factor- 23 concentrations in healthy men. J Clin Endocrinol Metab 91(8):3144-3149

Publisher's note Springer Nature remains neutral with regard to jurisdictional claims in published maps and institutional affiliations. 